\title{
Corticosteroids in Oral and Maxillofacial Lesions - A Review
}

\author{
Siccandar Jeelani* \\ Department of Oral Medicine and Radiology, Sri Venkateshwara Dental College, India
}

*Corresponding author: Siccandar Jeelani, Department of Oral Medicine and Radiology, Sri Venkateshwara Dental College, Ariyur, Puducherry, India

\begin{abstract}
Corticosteroids are used in the management of Oral and maxillofacial lesions because of their anti-inflammatory and immunosuppressive effects. The anti-inflammatory and immunosuppressive effects of steroids reflect them as the magic drug of choice. However, the potential risks have to be taken into judicious consideration before prescribing corticosteroids because they are a double-edged sword.
\end{abstract}

Keywords: Corticosteroids; Lichen planus; Oral sub mucous fibrosis; Recurrent aphthous stomatitis

\section{Introduction}

Corticosteroids are used in the management of Oral and maxillofacial lesions because of their anti-inflammatory and immunosuppressive effects. As immunity and immunosuppression work together in body defense, the anti-inflammatory and immunosuppressive effects of steroids together regulate these defense reactions and they remain as the magic therapy, however their administration has to be done judiciously weighing their benefits and adverse effects.

\section{Classification of Steroids}

\section{Glucocorticoids}

a. Short acting: Hydrocortisone, Cortisone

b. Intermediate acting: Prednisone, Prednisolone, Methylprednisolone, Triamcinolone

c. Long acting: Betamethasone, Dexamethasone, Paramethasone

Mineralocorticoids: Fludrocortisone, Deoxycorticosterone

\section{Applications of Steroids in Oral and Maxillofacial Lesions}

The therapeutic applications of steroids in oral and maxillofacial lesions are multifarious. This article reflects a few such pathologies which includes the following.

a) Lichen Planus

b) Oral Submucous fibrosis

c) Recurrent apthous Stomatitis

d) Pemphigus Vulgaris

e) Bell's Palsy

f) Mucocoele

g) Ramsay Hunt syndrome

h) Temperomandibular Joint Pain and dysfunction

i) Chronic ulcerative stomatitis \& Pyostomatitis vegetans 
j) Vesicular ulcerative and erosive lesion \& viral infections Erythema Multiforme, Herpes Virus infection \& Psoriasis

\section{Lichen planus}

Oral Lichen Planus is a common chronic immunologic inflammatory mucocutaneeous disorder that varies in appearance from keratotic (reticular or plaque like) to erythematous and ulcerative. Several studies were conducted with regard to the application of steroids in the management of Oral Lichen Planus.

a) In a study conducted by Zegarelli DJ - topical, injectable and systemic steroids were tried in 20 patients with 1 or more intraoral erosive or ulcerative lesions. Topical - Kenolog in Orabase (Triamcinolone acetonide) was applied to the affected sites as a thin film, four times daily ie, after every meal and before sleep for atleast 3 weeks. Injectable - During the same period the lesions were injected with Methyl Prednisolone once a week for 1- 3 weeks as per severity of lesion and response of the tissues. Systemic Prednisone was administered systemically as $30 \mathrm{mg} /$ day - $1^{\text {st }}$ week; $15 \mathrm{mg} /$ day $-2^{\text {nd }}$ week; $5 \mathrm{mg} /$ day $-3^{\text {rd }} \& 4^{\text {th }}$ week

b) In the above study 7 patients received topical \& injectable regimen, 5 followed topical \& systemic and 8 received all three. And it was found that 5 of 7 who received topical \& injectable showed complete improvement; 1 showed $75 \%$ improvement and 1 showed $25 \%$ improvement. In another study conducted by Lu S, Chen WJ, Eng HL - a combination of Prednisolone and levamisole was used in 23 patients. Levamisole 50mg thrice daily and Prednisolone $5 \mathrm{mg}$ thrice daily for 3 consecutive days per week were administered. It was found that almost all exhibited a dramatic response after the whole treatment course of 4-6 weeks and remained completely symptom free from 6-9 months without any medication. Also, there was diminution of the side effects of long-term high dosage prednisolone [1].

\section{Oral Submucous Fibrosis}

It is a slowly progressive chronic fibrotic disease of the oral cavity and oropharynx, characterized by fibro elastic change and inflammation of the mucosa, leading to a progressive inability to open the mouth, swallow or speak. Studies were conducted using steroids in the management of OSMF. In a study conducted by Gupta D, Sharma SC - a new treatment regimen using local injections consisting of a combination of Dexamethasone, Hyaluronidase and Chymotrypsin biweekly for 10 days was used. Good results were obtained in patients with severe limitation of opening and fibrotic bands with $74 \%$ had relief of painful ulceration, $100 \%$ relief of burning sensation, $79.5 \%$ blanching of oral mucosa and $94 \%$ relief of limited mouth opening [2].

\section{Recurrent apthous stomatitis}

It is the most common recurrent oral mucosal ulcerative disease, yet it remains one of the least understood diseases of the oral cavity. Although the term Apthae refers simply to the presence of an otherwise undefined ulcer, the disease apthous stomatitis may appear as lesions localized to the oral mucosa or a component of Vesiculoulcerative disease involving multiple organ systems. Studies were conducted to evaluate the effects of Steroids in Apthous stomatitis. In a study conducted by Muzio LL et al. the effects of topical Corticosteroid therapy in association with agents that increased the adhesion of the active drug to the oral mucosa in 30 RAS patients were studied. The $1^{\text {st }}$ group received $0.05 \%$ Clobetasol propionate, $2^{\text {nd }}$ group applied the drug in an adhesive denture paste while the $3^{\text {rd }}$ group applied the drug in an oral analgesic paste. Each patient was examined on the days when symptoms were present. They observed that the drug reduced the healing time in most patients and more significantly in the groups treated with adhesive denture paste. They concluded that the use of Clobetasol propionate in association with a denture adhesive had greater level of effectiveness as compared to the use of medication alone or with benzocaine containing orabase [3].

\section{Pemphigus vulgaris}

It is a potentially life-threatening disease that causes blisters and erosions of the skin and mucous membranes. These epithelial lesions are a result of autoantibodies that react with desmosomal glycoproteins that are present on the cell surface of the keratinocytes. The immune reaction against these glycoproteins causes a loss of cell to cell adhesion resulting in the formation of intraepithelial bullae. Studies were conducted using steroids in the management of Pemphigus. In a study conducted by Mignogna MD, Muzio LL, Mignogna RE et al. [3] - 16 patients with Pemphigus vulgaris with age between 26-76 were studied. All the patients complained of symptoms such as oral pain, dysphagia and odynophagia because of extensive mucosal erosion. The protocol for the initial / induction phase of treatment consisted of systemic Deflazacort (120mg daily in a single morning dose) and topical corticosteroids. The initial dose of Deflazacort was maintained from 2-4 weeks especially in patients with severe disease. In addition, in unresponsive cases patients received adjunctive systemic medication which included azathioprine (50-100mg daily) or cyclophosphamide (50mg daily). For Gingival lesions Clobetasol .05\% in custom made applicator trays were used [4].

\section{Bells palsy}

Idiopathic (Bell's) facial palsy is a self- limiting non- fatal and spontaneously remitting disorder of acute onset due to nonsuppurative inflammation of the facial nerve within the stylomastoid foramen. The role of steroids in Bell's Palsy was studied by Papazian MR, Campbell JA \& Nabi S. They stated that if a patient is seen within 2-3 weeks of onset of symptoms, Prednisone in doses of $1 \mathrm{mg} / \mathrm{kg} / \mathrm{d}$ for 10-14 days with gradual taper was recommended as long as there are no medical contraindications. The hope is that steroids might speed the recovery time and prevent synkinesis [5].

\section{Mucocele}

Mucocele is a clinical term that describes swelling caused by the accumulation of saliva at the site of a traumatized or 
obstructed minor salivary gland duct. Steroids have been tried in the management of Mucocele. In a study conducted by Wilcox JX, Hickory JE, 14 cases of mucocele of lip were treated with steroids.

A small syringe with a half inch 26-gauge needle was used to inject Triamcinolone acetate $(0.5$ to $1.5 \mathrm{ml})$. In 12 cases there was no recurrence for periods of observation of 16-24 months. It was concluded that the mechanism of action steroid is unclear, apparently the injected steroid either causes pressure atrophy of the feeding gland or resolution of the associated inflammation or both [6].

\section{Ramsay hunt syndrome}

It is a rare form of the disease characterized by Bell's palsy, unilateral vesicles of the ear and vesicles of the oral mucosa. Steroids were used in the management of Ramsay Hunt Syndrome by Sweeney CJ, Gilden DH in his study along with Acyclovir. A 7-10day course of Famciclovir (500mg thrice daily) or Acyclovir (800mg - 5 times daily) as well as Oral Prednisone (60mg daily for 3-5 days) was used. It was found that they improved the outcome of recovery from Facial palsy [7].

\section{Temperomandibular joint pain and dysfunction}

It is a complex disorder with suggested hypothesis being occlusal disharmony and psychological distress. Steroids have been tried in its management by Wenneberg B et al. who used a mixture of $0.5 \mathrm{ml}$ betamethasone in a suspension of $6 \mathrm{mg}$ per $\mathrm{ml}$ ( Celestone bifas) and equal volume of lidocaine chloride anhydrate (xylocaine $10 \mathrm{mg}$ perml) was injected into the superior compartment of the TMJ three times with an interval of 1 week between treatment. It was found that the subjective symptoms as well as the clinical signs were significantly reduced at the follow up after 8 years. Also, the erosions of the bony articular margins of the TMJ was found to be remineralised [8].

\section{Chronic ulcerative stomatitis \& pyostomatitis vegetans}

Chronic ulcerative stomatitis is a recent entity which resembles Erosive Lichen Planus or Oral Discoid lupus erythematoses in its clinical and histological manifestations. The use of Steroids in its management was studied by Lorenzana ER, Rees TD, Glass M, Detweiler JG. Flucinonide $.05 \%$ topical gel 4 times daily on the affected sites for 3 weeks and then Betamethasone dipropionate $.05 \% 4$ times daily for 2 months showed significant relief with less pain and in case of recurrences reinitiation of therapy was suggested. Pyostomatitis vegetans is a purulent inflammation of the mouth characterized by deep tissue vegetating or proliferative lesions that undergo ulceration and suppuration. The use of steroids in its management was studied by Chan SWY et al. Systemic steroid Prednisolone 20mg for 2 days, followed by $15 \mathrm{mg}$ for 1 day and then $10 \mathrm{mg}$ for 1 day and lastly $5 \mathrm{mg}$ for 1 day was used. Also, it was supplemented with a tetracycline mouthwash. A year later there was exacerbation of the lesions which was managed with Sulfasalazine [9].

\section{Vesiculo ulcerative and erosive lesion\& viral infections}

\section{a) Erythema multiforme}

It is an acute inflammatory disease of the skin and mucous membranes that causes a variety of skin lesions hence the name multiforme. The oral lesions typically inflammation accompanied by rapidly rupturing vesicles and bullae are often an important component of the clinical picture and are occasionally the only component. severe life threatening forms that may present as either Steven Johnson syndrome or Toxic epidermal necrolysis. Steroiods were used by Lozada F, in the management of Erythema multiforme. In a group of 12 patients the synergestic effect of Prednisone and Azathioprine were studied. The minimum effective dose of Prednisone when used alone was $40 \mathrm{mg}$ daily whereas with Azathioprine was never more than $25 \mathrm{mg}$ daily and ranged as low as $5 \mathrm{mg}$ daily. This study indicated that Azathioprine effectively reduced Corticosteroid requirement allowing lower doses of Prednisone with satisfactory clinical efficacy and a marked reduction in side effects.

\section{b) Herpes virus infections}

Varicella Zoster virus causes 2 distinct infections - a primary infection, Varicella (chickenpox) and a recurrent infection, Herpes zoster (shingles). A potential sequelae of Herpes zoster is Post Herpetic Neuralgia resulting from scarring of the involved nerve during infection. It is a painful sometimes debilitating condition that can last from months to years after the lesions are healed.

The role of Steroids in the management of Post Herpetic Neuralgia was studied by Whitley RJ, Weiss $\mathrm{H}$ et al. They considered 208 immunocompetent patients above the age of 50 and used the following regimen. Acyclovir $800 \mathrm{mg}$ - 5 times daily for 21 days and Prednisone $60 \mathrm{mg}$ per day for $1^{\text {st }} 7$ days; $30 \mathrm{mg}$ for next 7 days and $15 \mathrm{mg}$ per day for last 7 days. It was found that combined acyclovir and prednisone therapy can improve quality of life in older age group patients who are immune competent

\section{c) Psoriasis}

Psoriasis is a relatively common chronic dermatological disease characterized by epithelial hyperplasia presenting clinically as cutaneous erythematous papules and plaques covered by whitish scales commonly observed on the extensor-dorsal cutaneous surfaces. Although the etiology remains unclear, the epithelial changes are related to a defect in the control of keratinocyte proliferation. Steroids in Psoriasis of lip was tried in a study by Younai FS and Phelan JA. They used topical Fluocinonide (Lidex). $05 \%$ ointment mixed in equal parts with orabase four times daily over the lesion in addition to erythromycin $250 \mathrm{mg}$ four times daily for 5 days. Examination after 5 days showed complete resolution of all the lesions. The lip lesions healed with slight pigmentation remaining in the area and no evidence of pustules was present [10]. 


\section{Conclusion}

Overall corticosteroids are extremely beneficial in the treatment of oral and maxillofacial lesions. Both topical and systemic corticosteroids are used based on their indications. The dosage, duration of therapy, potential risks have to be taken into judicious consideration before prescribing corticosteroids. Ultimately steroids serve as a double-edged sword.

\section{References}

1. Zegarelli DJ (1983) Multimodality steroid therapy of Erosive and ulcerative Oral Lichen Planus. J Oral Med 38(3): 127-130.

2. Gupta D, Sharma SC (1988) Oral submucous Fibrosis - a new treatment regimen. J Oral maxillofac surg 46: 830-833.

3. Muzio LL, Valle AD, Mignogna MD, Sciubba J, Bucci E, et al. (2001) The treatment of oral apthous ulceration or erosive Lichen Planus with topical Clobetasol propionate three preparations: a clinical and pilot study on 54 patients. J Oral Path Med 30: 611-617.
4. https://onlinelibrary.wiley.com/doi/abs/10.1034/j.16000714.2000.290401.x

5. Mualichar M, Raghavan MRV, Bailoor DN (1987) Bilateral Bell's palsy: current concepts in aetiology and treatment. Case report. Aust Dent J 32(6): 412-416.

6. Wilcox JW, Hickory JE (1979) Nonsurgical resolution of Mucocele. J Oral Surg 6: 478 .

7. Sweeney CJ, Gilden DH (2001) Ramsay Hunt syndrome. J Neurol Neurosurg Psychiatry 71(2): 149-154.

8. Kopps S, Carlsson G, Haraldson T (1987) Long term effect of intra articular injections of sodium hyaluronate and corticosteroid on temperomandibular joint arthritis. J Oral Maxillofac surg 45: 929-935.

9. Lorenzana ER, Rees TD, Glass M (2000) Chronic ulcerative stomatitis: a case report. J Period 7(1): 104-111.

10. Lozada F (1981) Prednisone and Azathioprine in the treatment of patients with vesiculoreosive oral diseases. Oral Surgery 5(3): 257.

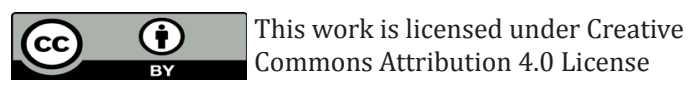

To Submit Your Article Click Here: Submit Article

DOI: 10.32474 /GJAPM.2019.01.000112

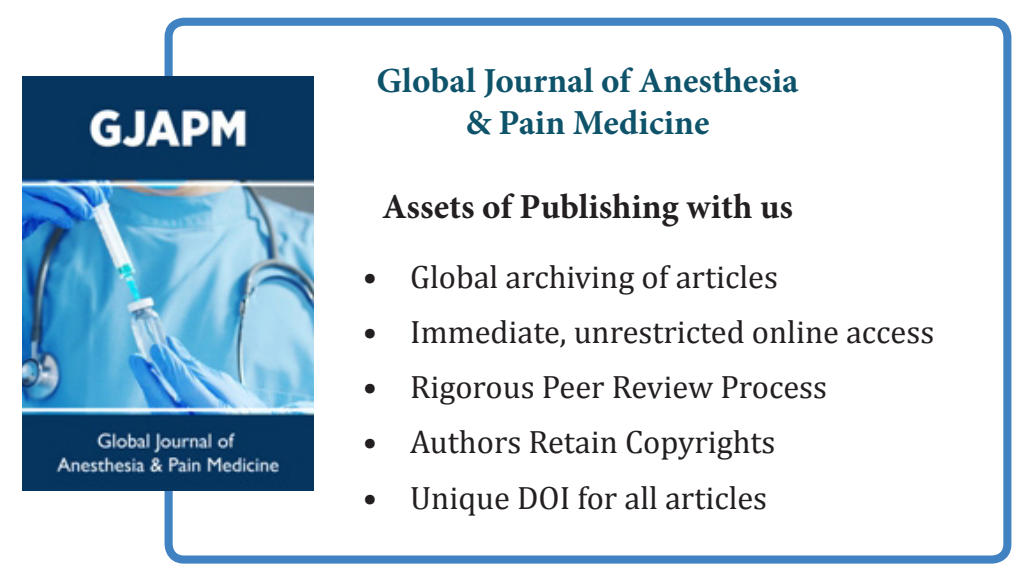

